Las didácticas específicas en tiempos de COVID-19

\title{
Universidad presencial confinada: Percepción de los estudiantes de Interpretación de la docencia presencial, semipresencial y no presencial durante la pandemia
}

\author{
Jessica PÉREZ-LUZARDO DÍAZ \\ Universidad de Las Palmas de Gran Canaria \\ jessica.perezluzardo@ulpgc.es \\ https://orcid.org/oooo-0002-2748-9892
}

Recepción: 21/05/2021 | Revisión: 02/09/2021 | Aceptación: 14/01/2022 | Publicación: 01/03/2022

\begin{abstract}
Resumen: Este estudio explora las adaptaciones didácticas en las asignaturas de Interpretación de los grados de Traducción e Interpretación y las preferencias de los estudiantes, así como las ventajas y desventajas percibidas por los estudiantes de la docencia presencial, semipresencial y a distancia en las asignaturas de la materia de Interpretación. El estudio parte de una muestra de 122 sujetos que cursaron asignaturas de interpretación desde que se declaró el confinamiento en marzo de 2020 hasta finales del primer semestre de 2021. La metodología empleada ha recurrido al uso de cuestionarios con preguntas cerradas, abiertas y de respuesta múltiple, por lo que reúne características de los estudios descriptivos mixtos. Los datos obtenidos ponen de manifiesto una preferencia por mantener la interacción directa con los docentes y compañeros incluso en un contexto de pandemia. Al tiempo que se produce cierto rechazo de las modalidades híbridas y a distancia por las dificultades técnicas y de conexión, se destacan las ventajas de poder evitar los desplazamientos y la mayor flexibilidad que estas ofrecen.
\end{abstract}

Palabras clave: interpretación; COVID-19; docencia a distancia; docencia semipresencial; didáctica de la interpretación.

\section{IN-PERSON UNIVERSITY CLASSES IN LOCKDOWN: INTERPRETING STUDENTS' PERCEP- TIONS OF IN-PERSON, BLENDED AND REMOTE TEACHING DURING THE PANDEMIC}

\begin{abstract}
This paper explores the adaptations to teaching interpreting subjects for Interpreting and Translation degree courses and the students' preferences, as well as the advantages and disadvantages of in-person, blended and remote teaching perceived by students. The study is based on a sample of 122 individuals who were studying interpreting subjects from the time lockdown was declared in March 2020 until the end of the first term of 2021. Questionnaires with closed, open questions and multiple-choice responses were administered, thereby complying with the characteristics of descriptive studies. The data revealed a preference for direct interaction with lecturers and fellow students, even in the context of a pandemic. While hybrid and remote options were generally rejected because
\end{abstract}


of technical difficulties and connectivity issues, the advantages of not having to travel and resulting greater flexibility were foregrounded.

Keywords: interpreting; COVID-19; remote learning; blended learning; teaching interpreting.

\title{
UNIVERSITAT PRESENCIAL CONFINADA: PERCEPCIÓ DELS ESTUDIANTS D'INTERPRETACIÓ DE LA DOCÈNCIA PRESENCIAL, SEMIPRESENCIAL I NO PRESENCIAL DURANT LA PANDÈMIA
}

\begin{abstract}
Resum: Aquest estudi explora les adaptacions didàctiques en les assignatures d'Interpretació dels graus de Traducció $i$ Interpretació i les preferències dels estudiants, així com els avantatges i inconvenients percebuts pels estudiants de la docència presencial, semipresencial i a distància en les assignatures de la matèria d'Interpretació. L'estudi parteix d'una mostra de 122 estudiants d'assignatures d'interpretació des que es va declarar el confinament el març de 2020 fins a finals del primer semestre de 2021. Es van utilitzar qüestionaris amb preguntes tancades, obertes i de resposta múltiple, complint així les característiques dels estudis descriptius mixtos. Les dades obtingudes posen de manifest la preferència de mantenir la interacció directa amb els docents $i$ companys, fins $i$ tot en un context de pandèmia. Alhora que se sent un cert rebuig per les modalitats híbrides i a distància per les dificultats tècniques i de connexió, es destaquen els avantatges de poder evitar els desplaçaments $i$ la major flexibilitat que ofereixen aquestes modalitats.
\end{abstract}

Paraules clau: interpretació; COVID-19; docència a distància; docència semipresencial; didàctica de la interpretació

\section{Introducción}

"El futuro de la[s] universidades españolas: la revolución digital ha venido para quedarse" (Vicioso, 7 de mayo de 2020), "Internet a pedales en la era virtual" (Florido, 9 de junio de 2020) son titulares de tiempos de inicio de la pandemia causada por el virus SARS-CoV-2 durante los que, de la noche a la mañana, la enseñanza universitaria se trasladó del aula al campus virtual. Los aspectos técnicos se intentaron solventar con ayudas a los estudiantes a través de las iniciativas de los vicerrectorados de estudiantes por facilitar dispositivos electrónicos a aquellos que no los tenían, mediante el préstamo de equipos informáticos, tabletas y ordenadores portátiles, por ejemplo (Silió, 23 de abril de 2020). Otros problemas, como la falta de conexión a internet y la mala cobertura, ocuparon los artículos de opinión y se denunciaron desde las asociaciones de vecinos de afectados y a través de la prensa (véase p. ej. El País Economía, 2020; Maese, 2020).

En este trabajo nos vamos a detener en cómo perciben los estudiantes las fórmulas adoptadas por la Facultad de Traducción e Interpretación (FTI) de la Universidad de Las Palmas de Gran Canaria (ULPGC) para la docencia de las asignaturas de las materias de interpretación desde marzo de 2020 y hasta la fecha. A partir de la quinta semana de docencia del segundo semestre del curso académico de 2019-2020 y hasta el 21 de junio de 2020, España permaneció confinada y se suspendió la docencia presencial en todo el territorio nacional, también en el archipiélago canario. Esto afectó a varias asignaturas de Interpretación del segundo ciclo de los grados y dobles titulaciones correspondientes. El escenario durante el curso 2020-2021 cambió, ya que desde la ULPGC y desde la FTI se hizo un esfuerzo para garantizar la mayor 
presencialidad posible, respetando siempre las medidas de seguridad necesarias para evitar contagios en las aulas y en las instalaciones universitarias.

Las soluciones adoptadas exigieron la colaboración e iniciativa de todos los estamentos de la Universidad. Así fue posible habilitar nuevos espacios para la docencia, establecer nuevos aforos en las aulas y crear aulas espejo. Esta solución híbrida permite que una parte del estudiantado acuda a clase con el profesor y que la sesión se retransmita. Dicha retransmisión se puede seguir desde casa (no presencial) y desde un espacio donde se proyecta la clase, el aula espejo. Esto se ha visto acompañado de otras acciones imprescindibles: la instalación de cámaras, micrófonos, dispensadores de desinfectante de manos y papel, el refuerzo del servicio de limpieza, así como la limitación del acceso y la señalización de los edificios para evitar circulación cruzada, así como la ventilación constante de las aulas. El resultado ha sido una combinación de presencialidad, semipresencialidad con aula espejo en el campus o en casa y -cuando no ha quedado más remedio- docencia no presencial.

El objetivo de este estudio es identificar las ventajas y los inconvenientes de la modalidad de presencialidad en las asignaturas de Interpretación desde la perspectiva de los estudiantes. Un objetivo secundario derivado del primero es averiguar qué tipo de semipresencial prefieren y por qué razón.

\section{Estado de la cuestión}

En los últimos años, y en los últimos meses con mayor intensidad, se ha producido un boom en la realización de estudios que abordan la transición de la docencia presencial a la no presencial (Bao, 2020; Flores y Gago, 2020; Zawacki-Richter, 2020), así como las ventajas y los inconvenientes de la docencia semipresencial, la docencia combinada (blended classroom), la clase invertida (flipped classroom) y la docencia no presencial a través de, por ejemplo, cursos en línea masivos y abiertos (CEMA o MOOC, siglas en inglés) (Crawford et al., 2020; García-Planas y Taberna Torres, 2021; Jaggars, 2021; Karalis y Raikou, 2020; Vladova et al., 2021). En el segundo semestre de 2020 se crearon espacios en redes sociales y en las propias páginas web de las universidades a las que acudían los docentes de programas de docencia universitaria presencial que se habían trasladado a la enseñanza a distancia sin margen de maniobra. En estos foros se intercambiaban experiencias de éxito y de fracaso, se sugerían alternativas y, sobre todo, se prestaba apoyo a los docentes (Crawford et al., 2020; Eldredge, s.f.; Llorens Largo, 2020; Universitat Oberta de Catalunya, s.f.). Esta tendencia se fue especializando y organizando por áreas de especialidad. También le llegó el momento a la docencia de la interpretación con Llewellyn Smith (s.f.) liderando un movimiento de asesoramiento desinteresado para la comunidad de docentes de Interpretación de grado y posgrado organizando eventos multitudinarios en los que invitaba a especialistas en la docencia a distancia de la interpretación que abordaban diversos aspectos, entre los cuales, cómo emplear las herramientas de videoconferencia para intentar simular las dinámicas habituales de ejercicios y cabinas de la docencia presencial.

Se debe recordar aquí que la docencia universitaria de la interpretación es joven. Es cierto que hay constancia de la existencia de programas de aprendizaje del oficio de intérprete muy anteriores (Rothman, 2015) y de su ejercicio profesional desde el principio de las civilizaciones (Kurz, 1985, 1986). Pero la profesión de intérprete de conferencias la ejercieron sobre todo personas que, durante su vida, casi de manera casual, habían ido adquiriendo las competencias 
necesarias para ejercerla (Baigorri Jalón, 2000). Solo a partir de mediados del siglo pasado comienza a formarse intérpretes de conferencias y surgen las primeras asociaciones de intérpretes internacionales (ibídem). No sería hasta 1972 cuando comienza la formación universitaria en España (Universidad Autónoma de Barcelona), seguida de la Universidad de Granada (1979) y la antigua Universidad Politécnica de Canarias (1988, actual ULPGC). En la actualidad, salvo dos excepciones (Universitat Oberta de Catalunya y Universidad Internacional de Valencia), los títulos de grado en Traducción e Interpretación se ofrecen en modalidad presencial.

Los retos que implican el estudio de la interpretación consecutiva y simultánea en modalidad no presencial o híbrida están relacionados con la naturaleza oral y comunicativa de la propia actividad y, en particular, con aspectos técnicos. En la actualidad las Facultades de Traducción e Interpretación disponen de laboratorios similares a los laboratorios de idiomas, pero con software diseñado para la enseñanza de la interpretación. Aparte se dispone de cabinas insonorizadas que simulan a las cabinas fijas o portátiles de los eventos con interpretación simultánea presencial y en algunos centros también se dispone de mesa de delegados para estudiantes oyentes o para interpretación consecutiva o susurrada (equipada con consolas con el software de interpretación). Desde el puesto del equipo docente, es posible seleccionar el material con el que se va a trabajar, grabar y reproducir las prestaciones de los estudiantes y diseñar sesiones específicas de trabajo, interrumpir a un estudiante concreto para corregir errores mientras trabaja, comentar en grupo o de forma individual los aspectos relevantes del proceso formativo, etc.

Reproducir estas funciones en un entorno híbrido o no presencial sin formación previa por parte del equipo docente, entraña cierta dificultad. Las aplicaciones disponibles en marzo de 2020 estaban concebidas para el apoyo a la presencial, pero no para impartirla en modalidad no presencial o híbrida. En el caso de la FTI de la ULPGC, el profesorado disponía en un primer momento de la plataforma Moodle y BigBlueBottom (sistema de conferencia de código abierto). Este sistema, en este caso particular, no permitía la grabación y recuperación de los ejercicios, no existía manera de simular tantos grupos pequeños de trabajo (en breakout rooms o aulas paralelas) como era necesario en las clases de interpretación consecutiva, dado el número de participantes. El docente no podía supervisar los grupos y asegurarse de que realmente estaban realizando los ejercicios sin dificultades técnicas. Además, el sistema se colapsó en repetidas ocasiones, por lo que las sesiones se veían interrumpidas. Con el paso de las semanas se habilitó Teams de Microsoft Office como alternativa, que ofrecía unas ventajas específicas, pero era una aplicación nueva para todos, profesorado y estudiantado. Las dificultades técnicas como ya se ha comentado también estaban en los hogares de los propios estudiantes que debían grabar sus ejercicios y subirlos al Moodle para su evaluación, disponer de un entorno con aislamiento acústico, al menos un dispositivo electrónico principal (ordenador, tableta), micrófono y auriculares, conexión a internet suficiente para trabajar con archivos de audio y vídeo, etc.

En los siguientes apartados abordaremos aquellos aspectos más destacados sobre los estudios de la docencia presencial, semipresencial y no presencial consultados. A partir de la revisión bibliográfica, se diseña un cuestionario para indagar acerca de las ventajas e inconvenientes de las distintas modalidades de enseñanza desde la perspectiva del estudiantado. El principal objetivo del estudio es averiguar cuál es la modalidad preferida por los estudiantes en las asignaturas de Interpretación e identificar los principales motivos que influyen en su elección. 


\subsection{Docencia presencial: ventajas e inconvenientes percibidas por estudiantes}

Si bien la enseñanza universitaria sigue siendo en su mayoría presencial, siguiendo la tradición de siglos de existencia, la mayoría de las universidades cuentan ya con campus virtuales en los que comparten materiales y tareas no solo para estudiantes de programas de teleformación, sino también para estudiantes que cursan programas presenciales. Estas aulas virtuales sirven de apoyo a las clases presenciales, complementando las opciones en el proceso de enseñanzaaprendizaje y las sesiones presenciales (Martínez, 2017; Popa et al., 2020).

No es de extrañar que ya desde hace años algunos expertos vaticinen el declive de la docencia universitaria presencial tradicional (Boettchar y Conrad, 2016). Con todo, lo cierto es que aún es posible encontrar estudios que ponen de manifiesto que algunos estudiantes universitarios prefieren las clases presenciales a las clases no presenciales (Jaggars, 2014; Meulenbroeks, 2020; Romero Andonegui et al., 2021). En estos casos, los estudiantes perciben que la interacción tanto entre iguales como con sus docentes es más rápida y ponen en valor el contacto visual y la importancia de la comunicación no verbal (Meulenbroeks, 2020). En esta fórmula de docencia, el docente puede supervisar que la relación del estudiante con el contenido, con otros estudiantes y con el cuerpo docente esté equilibrada (Knowles et al., 2005). Esta ventaja comparativa de la docencia presencial supone un reto de la docencia en formatos híbridos y no presenciales en muchos centros universitarios.

La docencia presencial también tiene sus limitaciones. Quizá la principal sea que requiere que el docente y los estudiantes estén presentes en la misma aula. Los partidarios de las opciones semipresenciales o no presenciales critican la rigidez del formato que está sujeto a un horario y, por tanto, no aporta flexibilidad a los participantes (Martínez, 2017). Otro inconveniente destacado se asocia al modelo clásico de la clase magistral, durante la cual el docente expone contenido a estudiantes que no pueden aprovechar del todo la clase, porque no están preparados (Lorenzetti, 2004).

\subsection{Docencia semipresencial y no presencial: ventajas e inconvenientes percibidas por estudiantes}

La docencia no presencial ofrece grandes oportunidades a las universidades en un contexto global en el que se aspira a atraer a estudiantes de distinta procedencia, pero sin aumentar la plantilla de profesores ni disponer de nuevos espacios docentes (Lorenzetti, 2004). De hecho, son ya una mayoría las instituciones universitarias que cuentan en sus planes estratégicos con una oferta no presencial a corto, medio y largo plazo (Bowers y Kuman, 2015; Martínez, 2017) o que ya la han desarrollado.

A pesar de que hay estudios que parecen indicar unas tasas de abandono superiores en este tipo de opciones de enseñanza (Boston et al., 2011; Bowers y Kumar, 2015), también hay estudios que confirman que los estudiantes reciben bien las propuestas docentes que combinan sesiones presenciales con trabajo no presencial (Plota y Karalis, 2019). La enseñanza combinada, entendida como un método que "combines face-to-face instruction with computer-mediated instruction" (Graham, 2006, p. 5), es una de las opciones de docencia semipresencial más extendida. En realidad, esta descripción no refleja bien la combinación de teorías, métodos y tecnologías para optimizar el aprendizaje en un contexto dado (Cronje, 2020). Pero la idea es extraer lo mejor de ambas opciones, ofreciendo a los estudiantes mucha autonomía para que 
aborden el contenido a su ritmo, aprovechando las interacciones presenciales para optimizar el contacto del estudiante con sus compañeros y con el docente. Así lo detecta Sorokova (2020), quien constata además mejores resultados académicos en un grupo con docencia combinada, frente a los estudiantes de un grupo con docencia solo presencial. Por su parte, Arıf y Omar (2019) confirman una mayor motivación en los estudiantes que participan en cursos que incluyen una metodología con clases invertidas. Otra ventaja destacada del formato híbrido es que ofrece la oportunidad al estudiantado de familiarizarse con el material antes de entrar en el aula (Lorenzetti, 2004). De esta manera, los docentes pueden profundizar en el contenido en el aula en las sesiones presenciales, si bien requiere un diseño exhaustivo de las tareas que se realizan en modalidad presencial y no presencial (ibíd.) para optimizar las ventajas de cada opción.

Como era de esperar, es posible encontrar algún estudio que apunta a que los estudiantes señalan como un inconveniente la falta de flexibilidad en las programaciones de los formatos híbridos (El Mansour y Mupinga, 2007) frente a las opciones a distancia, y también estudios que muestran la preferencia de los estudiantes de formatos híbridos, frente a la opción exclusivamente online (Sousa Santos et al., 2017). Esto podría depender del porcentaje de contenido que se imparte en formato no presencial. Boettcher y Conrad (2016) distinguen entre aquellos cursos ligeramente híbridos con un porcentaje de 15 a 39 de contenido en línea frente a la modalidad de curso híbrida con un porcentaje mayor que oscila entre el 40 y 79 por ciento.

La docencia no presencial, por su parte, es aquella en la que el equipo docente y los estudiantes no comparten espacio en ningún momento durante la formación. En algunos casos, solo coinciden durante las pruebas de evaluación final (es el caso de la Universidad Nacional de Educación a Distancia, por ejemplo). Ni siquiera requiere que el docente interactúe con los estudiantes en sesiones síncronas de manera habitual. Para el diálogo se recurre a conversaciones en foros o en chats individuales o por grupos. De ahí la importancia de que la propuesta docente tenga en cuenta estrategias de apoyo y motivación para los estudiantes que reemplacen el contacto directo de las sesiones presenciales (Bennett y Lockyer, 2004) para frenar las tasas de abandono (Martínez, 2017).

Como requisito de partida para la docencia no presencial se deben garantizar unas condiciones mínimas (Welch y Napoleon, 2015): una conexión a internet suficiente (estable), equipo básico (ordenador y periféricos), la formación especializada de los docentes, así como su buena disposición.

Según estudios comparativos de calidad para contrastar ofertas no presenciales con sus equivalentes presenciales, parece ser que su calidad es muy similar ( $a p$. Bowers y Kumar, 2015). Este tipo de docencia "requires the development of technical skills and practical strategies" (Bennet y Lockyer, 2004, p. 242) por parte de los docentes y los estudiantes. Para ello es fundamental contar con la colaboración de la institución en su conjunto, tanto en el programa de formación del profesorado, como en el desarrollo de herramientas suficientes que permitan la docencia no presencial (Caron, 2013). No es suficiente con que el docente se forme y tenga una excelente predisposición: se hace necesaria una labor de acompañamiento constante. Cuando esto sucede y los docentes planifican con atención la interacción de los estudiantes con el contenido, el propio equipo docente y otros compañeros, los estudiantes pueden tener incluso mayor interacción y la participación puede estar mejor distribuida que en la docencia presencial al emplear diferentes recursos síncronos y asíncronos para conversar y comunicar (Bowers y Kumar, 2015; Northrup, 2002). Young (2006) observa en su estudio que la docencia no presencial es eficaz cuando los profesores "remain visibly and actively involved 
in the learning, maybe even to a greater dregree than in the traditional classroom" (p. 74), lo que requiere sin duda un esfuerzo adicional por parte del docente.

A la hora de diseñar un curso o una asignatura semipresencial o no presencial, habría que tener presente tanto las ventajas como los inconvenientes manifestados por los estudiantes (Wang et al., 2019). Una de las ventajas más aclamadas de los formatos no presenciales es la flexibilidad que pueden ofrecer para que los estudiantes organicen su tiempo (Barak et al., 2016; Bowers y Kumar, 2015; Caron, 2013; Northrup, 2002; Young, 2006), sobre todo aquellos que combinan sus estudios con otras obligaciones: trabajos a tiempo parcial, responsabilidades familiares, otros estudios, etc.

Entre los inconvenientes señalados por el estudiantado en estudios previos (Area-Moreira et al., 2020; Barak et al., 2016; Bowers y Kumar, 2015), cabe destacar la sensación de soledad, problemas de adaptación, problemas de autodisciplina para seguir el ritmo de trabajo necesario para cumplir con los objetivos de aprendizaje (aprendizaje autorregulado), así como problemas técnicos.

\section{Metodología 2.1 Diseño del estudio}

A partir de la revisión bibliográfica y de los estudios previos realizados (El Mansour y Mupinga, 2007; Popa et al., 2020; Sorokova, 2020), se diseñó un cuestionario semiestructurado con preguntas cerradas, de respuesta múltiple y otras de respuesta abierta que aborda las ventajas e inconvenientes de cada una de las modalidades: no presencial, semipresencial y no presencial, siguiendo las recomendaciones metodológicas de Dewaele (2018). El cuestionario incluye cinco preguntas para la descripción de la muestra: género y edad, título y curso que cursan, asignaturas de interpretación y modalidad de docencia recibida durante el periodo del estudio para cada asignatura. A continuación, hay 12 preguntas, de las cuales 10 son preguntas de elección múltiple y dos son preguntas abiertas. En estas doce preguntas se abordan las ventajas y los inconvenientes de la enseñanza presencial, semipresencial y no presencial en las asignaturas de interpretación. Una de las preguntas abiertas incide en los motivos por los que los estudiantes prefieren el aula espejo en la propia Facultad o no presencial (en casa). Se añade una pregunta sobre su impresión acerca de la posibilidad de que la modalidad no presencial sustituya a la presencial en la enseñanza de interpretación y, por último, se anima a los participantes a exponer otras reflexiones sobre la modalidad de docencia y cómo ha influido o influye en su aprendizaje en las materias de interpretación (pregunta abierta).

Para facilitar el análisis de las respuestas de una pregunta abierta con un número elevado de respuestas, se procede a su categorización para poder clasificar la tipología de respuesta (Dörnyei, 2018). El análisis cualitativo de esta pregunta se realizó siguiendo cuatro pasos. En primer lugar, las respuestas de los estudiantes se reorganizaron en pequeñas unidades con ideas básicas. Cada unidad, junto al comentario original, se transfirió a una tabla y se asignaron códigos. En segundo lugar, una segunda lectura de los datos originales permitió identificar los patrones se respuesta, a partir de los cuales se agruparon aquellas respuestas que hacían referencia a aspectos similares. En cuarto lugar, se asignan códigos para cuantificar y clasificar mejor las respuestas obtenidas (Brown, 2001). 


\subsection{Descripción de la muestra}

La muestra total es de $\mathrm{N}=122$ estudiantes (96 mujeres) de la Facultad de Traducción e Interpretación de la Universidad de Las Palmas de Gran Canaria (ULPGC). Este estudio descriptivo se realizó durante el curso 2020-2021. La mayoría de los encuestados (95,8\%) tenía entre 22-25 años (115 del total). En todos los casos habían cursado una o más asignaturas de interpretación durante el periodo del estudio que va de marzo de 2020, fecha en la que se declaró el confinamiento domiciliario nacional en España, y febrero de 2021, lo que aborda tanto periodos de docencia y evaluación no presencial obligada (la docencia y la evaluación estaba programada en formato presencial en todas las asignaturas de interpretación), como soluciones presenciales y semipresenciales para el curso 2020-2021, ya que se evitó la docencia no presencial en esta materia.

Tras recibir el visto bueno del decanato, se envió una solicitud de colaboración a todos los estudiantes de tercero, cuarto y quinto de los grados, esto es, a un total de 186 estudiantes. El enlace al cuestionario estuvo disponible durante tres semanas y se envió un recordatorio a los quince días. Para la distribución de los cuestionarios entre los estudiantes de tercero, se contó con la colaboración de un docente que facilitó y animó a los estudiantes a participar durante una de sus sesiones de clase, recordando el carácter voluntario de la participación. Este hecho se traduce en una ligera mayor participación de los estudiantes de este curso $(58,7 \%)$. Cabe destacar que la mayoría de estos estudiantes no tenía docencia en asignaturas de interpretación durante el curso 2019-2020, por lo que no habían experimentado docencia no presencial en ninguna asignatura de interpretación, salvo los repetidores.

La única asignatura que se cursó de forma no presencial síncrona por los estudiantes de la muestra es Interpretación Consecutiva BII Inglés, asignatura del segundo semestre que coincidió con el periodo de confinamiento en 2020. En la asignatura de Interpretación Consecutiva C Francés tres estudiantes comentan haberla realizado de forma no presencial. Es posible que sean estudiantes con un perfil de riesgo, porque el resto cursó esta asignatura de forma presencial o semipresencial con aula espejo en la Facultad o en casa, al igual que la asignatura Interpretación Consecutiva C Alemán. La asignatura de Interpretación Consecutiva BI Inglés se cursó en modalidad presencial o semipresencial con aula espejo en casa o en la Facultad y la asignatura de Interpretación Simultánea B I Inglés se cursó en modalidad presencial en todos los casos del estudio (Tabla 1).

\begin{tabular}{lccccc}
\hline & Presencial & $\begin{array}{c}\text { Semipresenci } \\
\text { al con aula } \\
\text { espejo en la } \\
\text { FTI }\end{array}$ & $\begin{array}{c}\text { Semipresenci } \\
\text { al con aula } \\
\text { espejo desde } \\
\text { casa }\end{array}$ & No presencial & $\begin{array}{c}\text { No la he } \\
\text { cursado }\end{array}$ \\
\hline & $\mathrm{n}(\%)$ & $\mathrm{n}(\%)$ & $\mathrm{n}(\%)$ & $\mathrm{n}(\%)$ & $\mathrm{n}(\%)$ \\
\hline $\begin{array}{l}\text { Interpretación } \\
\text { Consecutiva BI } \\
\text { Inglés }\end{array}$ & $36(29,5)$ & $12(9,8)$ & $49(40,2)$ & $2(1,6)$ & $23(18,9)$ \\
\hline $\begin{array}{l}\text { Interpretación } \\
\text { Consecutiva BII } \\
\text { Inglés }\end{array}$ & $61(50)$ & $18(14,8)$ & $2(1,6)$ & $23(18,9)$ & $18(14,8)$ \\
\hline $\begin{array}{l}\text { Interpretación } \\
\text { Simultánea BI } \\
\text { Inglés }\end{array}$ & $43(35,2)$ & & & \\
\hline $\begin{array}{l}\text { Interpretación } \\
\text { Simultánea BII } \\
\text { Inglés }\end{array}$ & $39(32)$ & & & & \\
\hline
\end{tabular}




\begin{tabular}{|c|c|c|c|c|c|}
\hline $\begin{array}{l}\text { Interpretación } \\
\text { Consecutiva C } \\
\text { Alemán }^{b}\end{array}$ & $11(9)$ & $3(2,5)$ & $10(8,2)$ & & $98(80,3)$ \\
\hline $\begin{array}{l}\text { Interpretación } \\
\text { Consecutiva C } \\
\text { Francés }{ }^{b}\end{array}$ & $10(8,2)$ & $5(4,1)$ & $10(8,2)$ & $4(3,3)$ & $93(76,2)$ \\
\hline
\end{tabular}

Tabla 1. Frecuencias de las asignaturas cursadas y la modalidad.

\section{Resultados y discusión}

A continuación, se presentan los resultados obtenidos en el presente estudio. Se han organizado los resultados y su discusión siguiendo la estructura del cuestionario.

\subsection{Ventajas e inconvenientes de la modalidad presencial}

Tal y como se aprecia en la Tabla 2, ningún encuestado considera que la enseñanza presencial no tenga alguna ventaja. Las ventajas más destacadas son la "interacción con el docente y la resolución rápida de dudas" y "el feedback directo e inmediato del docente". Ambas ventajas fueron destacadas por 101 sujetos, lo que supone el $84,2 \%$ de la muestra. Además, el $56,7 \%$ de la muestra (68 sujetos) resalta la "interacción directa con sus compañeros" como otra de las ventajas de la modalidad presencial. Otra opción seleccionada por el $71,7 \%$ de la muestra ( 86 sujetos) refuerza la noción de que la enseñanza presencial favorece la capacidad de atención.

\begin{tabular}{|c|c|c|c|}
\hline & & \multicolumn{2}{|c|}{$\mathrm{n}(\%)$} \\
\hline & & Seleccionado & $\begin{array}{c}\text { No } \\
\text { seleccionado }\end{array}$ \\
\hline \multirow{8}{*}{$\begin{array}{l}\text { Ventajas } \\
\text { modalidad } \\
\text { presencial }\end{array}$} & La enseñanza presencial no tiene ninguna ventaja & 0 & $122(100)$ \\
\hline & $\begin{array}{l}\text { La enseñanza presencial no requiere ni depende } \\
\text { tanto del equipamiento informático }\end{array}$ & $38(31,1)$ & $84(68,9)$ \\
\hline & $\begin{array}{l}\text { La enseñanza presencial no depende tanto de la } \\
\text { conexión a internet/wifi }\end{array}$ & $60(49,2)$ & $62(50,8)$ \\
\hline & $\begin{array}{l}\text { En la enseñanza presencial hay interacción con el } \\
\text { docente (resolución rápida de dudas) }\end{array}$ & $102(83,6)$ & $20(16,4)$ \\
\hline & $\begin{array}{l}\text { En la enseñanza presencial hay más interacción } \\
\text { directa con los compañeros }\end{array}$ & $69(56,6)$ & $53(43,4)$ \\
\hline & $\begin{array}{l}\text { En la enseñanza presencial hay feedback directo e } \\
\text { inmediato del docente }\end{array}$ & $102(83,6)$ & $20(16,4)$ \\
\hline & $\begin{array}{l}\text { La enseñanza presencial favorece mi capacidad de } \\
\text { atención }\end{array}$ & $87(71,3)$ & $35(28,7)$ \\
\hline & $\begin{array}{l}\text { En la enseñanza presencial hay horarios fijos } \\
\text { (trabajo diario y estructurado) }\end{array}$ & $65(53,3)$ & $57(46,7)$ \\
\hline \multirow{9}{*}{$\begin{array}{l}\text { Inconvenientes } \\
\text { modalidad } \\
\text { presencial }\end{array}$} & $\begin{array}{l}\text { La enseñanza presencial no tiene ningún } \\
\text { inconveniente }\end{array}$ & $41(33,6)$ & $81(66,4)$ \\
\hline & $\begin{array}{l}\text { Los horarios fijos de la enseñanza presencial } \\
\text { (dificultad para compaginar con el trabajo y } \\
\text { familia) }\end{array}$ & $20(16,4)$ & $102(83,6)$ \\
\hline & $\begin{array}{l}\text { La enseñanza presencial no requiere ni emplea } \\
\text { tantos recursos alternativos }\end{array}$ & $11(9,0)$ & $111(91)$ \\
\hline & Hay que sumar los gastos del desplazamiento & $69(56,6)$ & $53(43,5)$ \\
\hline & Implica interacción directa con el docente & $4(3,3)$ & $118(96,7)$ \\
\hline & Implica interacción directa con los compañeros & $8(6,6)$ & $114(93,4)$ \\
\hline & $\begin{array}{l}\text { En la enseñanza presencial hay feedback directo } \\
\text { del docente delante de los compañeros }\end{array}$ & $11(9,0)$ & $111(91,0)$ \\
\hline & $\begin{array}{l}\text { La enseñanza presencial satura mi capacidad de } \\
\text { atención }\end{array}$ & $16(13,1)$ & $106(86,9)$ \\
\hline & Total & 122 & 100 \\
\hline
\end{tabular}

Tabla 2. Frecuencias sobre las ventajas e inconvenientes de la modalidad presencial en las asignaturas de interpretación. 
Con respecto a los inconvenientes de la enseñanza presencial en las asignaturas de interpretación cabe señalar que una tercera parte de la muestra considera que no tiene ningún inconveniente. La desventaja con mayor apoyo es la relativa a los gastos de desplazamiento $(58,5 \%)$. Solo 4 sujetos seleccionan como desventaja la interacción directa con el docente y tan solo 8 la interacción directa con los compañeros.

Resulta significativo que ningún encuestado considere que la enseñanza presencial no tenga alguna ventaja, porque en un contexto de pandemia era de esperar que los estudiantes mostrasen cierto rechazo hacia la enseñanza presencial. Este resultado refuerza las observaciones del estudio previo de Meulenbrocks (2020) que señalaba una preferencia de la presencialidad incluso con las normas de distanciamiento social propias del momento del estudio y con independencia de que en el ámbito privado los estudiantes se desenvuelvan mejor con la tecnología (Romero Andonegui et al., 2021). De igual forma, y al contrario de lo que cabía esperar, la mayoría no marca como inconveniente de la modalidad presencial la falta de flexibilidad que supone tener unos horarios fijos para compaginar los estudios con otras actividades.

Cabe señalar que ni los docentes ni los estudiantes estaban preparados para el cambio repentino de modalidad de enseñanza. En el caso concreto de las asignaturas de interpretación, la retroalimentación tanto de los docentes como de los compañeros es muy importante. La modalidad presencial facilita la posibilidad de trabajar en parejas, en grupos pequeños antes de pasar a los ejercicios ante toda la clase. Por otro lado, las instalaciones presenciales disponen de cabinas de interpretación que reúnen las condiciones adecuadas de insonorización y audio, lo que simula la experiencia profesional. Además, los aspectos técnicos (grabación, selección del material, control del volumen) recaen sobre el docente, permitiendo al estudiante centrarse sobre las competencias específicas de cada ejercicio. Dado que no hubo fase previa de entrenamiento ni de los estudiantes ni de los docentes, es posible que las respuestas se hayan visto afectadas por estos motivos.

\subsection{Ventajas e inconvenientes de la modalidad semipresencial}

Los estudiantes apuntan como mayor ventaja de la modalidad de enseñanza semipresencial la cierta libertad que ofrece, así como las posibilidades de conciliación (Barak et al., 2016; Bowers y Kumar, 2015; Caron, 2013). A esta ventaja le sigue de cerca la posibilidad de reducir el contacto social y los desplazamientos, que recibe el apoyo del 53,3\% (véase Tabla 3).

\begin{tabular}{|c|c|c|c|}
\hline & & & $(\%)$ \\
\hline & & Seleccionado & No seleccionado \\
\hline $\begin{array}{l}\text { Ventajas } \\
\text { modalidad }\end{array}$ & $\begin{array}{l}\text { La enseñanza semipresencial no tiene ninguna } \\
\text { ventaja }\end{array}$ & $21(17,2)$ & $101(82,8)$ \\
\hline semipresencial & $\begin{array}{l}\text { Este formato híbrido permite cierta libertad y } \\
\text { conciliación }\end{array}$ & $71(58,2)$ & $51(41,8)$ \\
\hline & $\begin{array}{l}\text { La enseñanza semipresencial implica mayor uso } \\
\text { de herramientas informáticas }\end{array}$ & $22(18)$ & $100(82)$ \\
\hline & $\begin{array}{l}\text { La enseñanza semipresencial implica el uso de } \\
\text { plataformas de videoconferencia }\end{array}$ & $16(13,1)$ & $106(86,9)$ \\
\hline & $\begin{array}{l}\text { La enseñanza semipresencial reduce el contacto } \\
\text { social y los desplazamientos }\end{array}$ & $64(52,5)$ & $58(47,5)$ \\
\hline & $\begin{array}{l}\text { La enseñanza semipresencial reduce el feedback } \\
\text { de los docentes (ante mis compañeros) }\end{array}$ & $9(7,4)$ & $113(92,6)$ \\
\hline $\begin{array}{l}\text { Inconvenientes } \\
\text { modalidad }\end{array}$ & $\begin{array}{l}\text { La enseñanza semipresencial no tiene ningún } \\
\text { inconveniente. }\end{array}$ & $5(4,1)$ & $117(95,9)$ \\
\hline semipresencial & $\begin{array}{l}\text { Falta de flexibilidad en el formato híbrido o } \\
\text { semipresencial }\end{array}$ & $24(19,7)$ & $98(80,3)$ \\
\hline & $\begin{array}{l}\text { En la enseñanza semipresencial surgen } \\
\text { dificultades técnicas con el uso de las } \\
\text { herramicntas informáticas }\end{array}$ & $78(63,9)$ & $44(36,1)$ \\
\hline
\end{tabular}




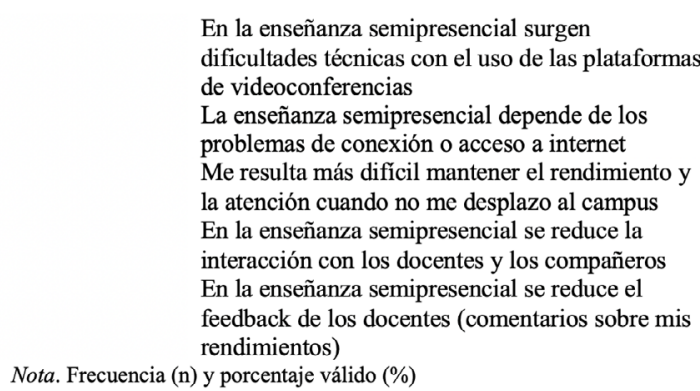

$\begin{array}{ll}90(73,8) & 32(26,2) \\ 90(73,8) & 32(26,2) \\ 53(43,4) & 69(56,6) \\ 72(59,0) & 50(41,0) \\ 72(59,0) & 50(41,0)\end{array}$

Tabla 3. Frecuencias de las ventajas e inconvenientes de la modalidad semipresencial en las asignaturas de interpretación.

Tal y como se observa en la Tabla 3, las respuestas son más numerosas a la hora de seleccionar inconvenientes de esta modalidad. El 74,2\% destaca que depende de los problemas de conexión y acceso a internet, así como que surgen dificultades con el uso de las plataformas de videoconferencia. También predominan las dificultades técnicas con el uso de las herramientas informáticas $(64,2 \%)$. Además, el 59,2\% señala como desventaja que "se reduce la interacción con los docentes y compañeros" y que "se reduce el feedback de los docentes (comentarios sobre el rendimiento)". Con respecto a la modalidad semipresencial, el $45 \%$ manifiesta que le "resulta más difícil mantener la atención" en las sesiones no presenciales. Solo cinco sujetos $(4,2 \%)$ señalan que esta modalidad no tiene ningún inconveniente.

Se obtiene el resultado esperado al observar el número de estudiantes que destaca como ventaja la cierta libertad que ofrece la modalidad semipresencial, así como las posibilidades de conciliación en la misma línea que estudios anteriores (Barak et al., 2016; Bowers y Kumar, 2015; Caron, 2013). Aun sumando el 58,2\% del total, llama la atención que la modalidad semipresencial no cuente con un apoyo más rotundo. Por otro lado, sorprende que se destaque la ventaja de evitar el contacto social, algo lógico en un contexto de pandemia, pero que contrasta con los resultados obtenidos para la modalidad presencial, donde se menciona como ventaja la mayor interacción con docentes y compañeros (véase Tabla 2).

Precisamente en los inconvenientes, un porcentaje también elevado destaca la menor interacción estudiante-docente y entre compañeros, como un problema, algo que recuerda al sentimiento de soledad y aislamiento detectado por El Mansour y Mupinga (2007). A la vista de las respuestas relativas a las incidencias, los problemas técnicos y de conexión referidos en este caso deberían continuar siendo objeto de estudio por parte las instituciones por si volviera a haber medidas que llevasen a la enseñanza virtual o semipresencial. Según la conferencia de rectores, en 2020 un 3\% del estudiantado universitario español, lo que equivale a unos 36.000, tenía problemas técnicos para seguir las clases (Silió, 23 de abril de 2020).

Por último, cabe destacar cierta correlación entre aquellos que comentan que en esta modalidad se concentran peor cuando las sesiones no son presenciales con el resultado obtenido en las ventajas de la modalidad presencial: el $71,3 \%$ de estudiantes afirma que se concentran más en las asignaturas de interpretación cuando estas son presenciales. 


\subsection{Ventajas e inconvenientes de la modalidad no presencial}

Se presentan a continuación las ventajas e inconvenientes destacados por los sujetos que sí habían cursado alguna asignatura de la materia de interpretación en modalidad no presencial. Cabe puntualizar que los estudiantes de la muestra cursaron asignaturas en modalidad no presencial síncrona.

Como podemos observar en la Tabla 4, las principales ventajas son que se evitan desplazamientos y que aporta mayor flexibilidad cuando la docencia no presencial es asíncrona. Salvo uno de los inconvenientes del listado, todos los ítems reciben un apoyo superior al $50 \%$ de la muestra, siendo las más apoyadas aquellas relacionadas con las dificultades técnicas del uso de las herramientas informáticas, las plataformas de videoconferencias y la dependencia de las conexiones a internet. Vuelve a resaltarse como inconveniente la falta de interacción directa con los docentes y los compañeros, que se puede decir que es una constante en el estudio para las modalidades semipresenciales y no presenciales.

$\mathrm{n}(\%)$

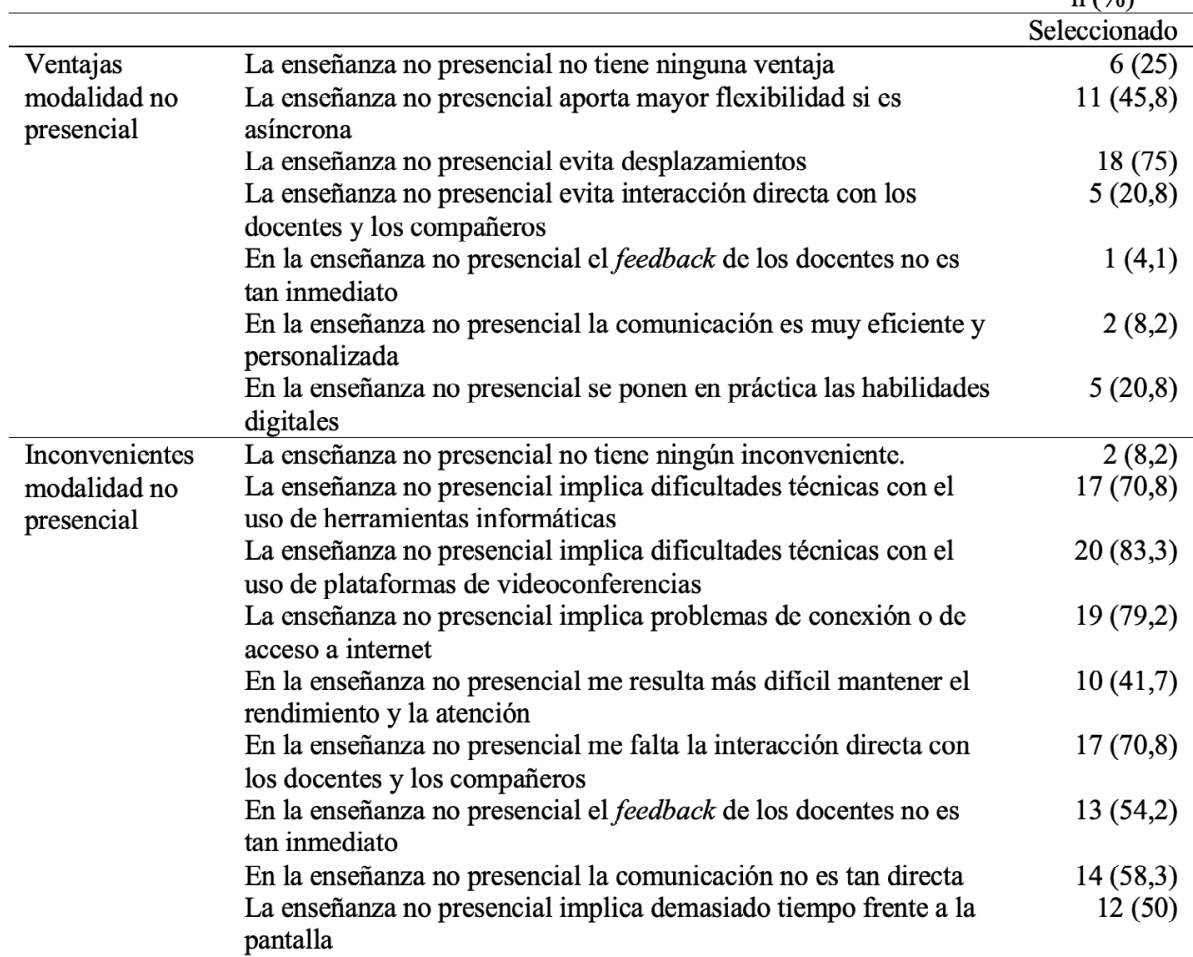

Tabla 4. Frecuencias de las ventajas e inconvenientes de la modalidad no presencial en las asignaturas de interpretación.

En el caso de las respuestas sobre la modalidad no presencial hay que tener en cuenta que su validez está limitada a la poca experiencia que los estudiantes han tenido con esta modalidad en las asignaturas de interpretación y que con toda seguridad están extrapolando su experiencia en otras materias, porque, si bien el número de estudiantes que afirma haber cursado una asignatura en modalidad no presencial es bajo en la muestra ( 24 sujetos, que corresponde al $19,5 \%$ del total), se obtuvo respuesta de 118 sujetos en las ventajas e 
inconvenientes de esta modalidad, aunque en la Tabla 4 sólo se recogen las respuestas de aquellos que asistieron a clases de interpretación no presencial. Aun teniendo en cuenta que el número de respuestas no permite hacer generalizaciones, es posible observar mayor peso en las respuestas que señalan los inconvenientes.

\subsection{Consideraciones finales sobre las ventajas e inconvenientes de las distintas modalidades}

Las preguntas que abordaban las ventajas e inconvenientes eran todas de respuesta múltiple. Resulta pertinente apuntar que, frente a las 516 ventajas seleccionadas por la muestra en la pregunta sobre la enseñanza presencial, se han seleccionado solo 182 ventajas de la modalidad semipresencial y 185 ventajas de la modalidad no presencial. Aún más ilustrativo es el número de respuestas total de inconvenientes seleccionado por la muestra: 139 presencial, 474 semipresencial y 558 no presencial o a distancia. Es conveniente recordar que este estudio se refiere en todo momento a la enseñanza de asignaturas de interpretación, lo que es posible que tenga un efecto sobre la clara preferencia de los estudiantes hacia la presencialidad. Incluso en un contexto complejo como el vivido durante el estudio. El riesgo percibido no supera el inconveniente que les plantea cursar estas asignaturas en modalidades híbridas o a distancia.

Cabe señalar también que no se han detectado diferencias de género en esta muestra de estudio, y las diferencias entre grados y cursos están supeditadas únicamente a la modalidad de enseñanza (presencial, semipresencial o no presencial).

\subsection{El aula espejo en la Facultad o desde casa en las soluciones híbridas o se- mipresenciales}

En el cuestionario se incluyó una pregunta sobre la preferencia de aula espejo en la propia facultad frente al aula espejo desde casa. En la Tabla 5 se observa que 102 de los 122 sujetos de la muestra prefieren el aula espejo desde casa $(83,6 \%$ de las respuestas obtenidas) en las soluciones híbridas o semipresenciales frente a solo 19 sujetos que se inclinan por acudir a ver la retransmisión desde la propia Facultad.

\begin{tabular}{lrr} 
& Frecuencia & Porcentaje válido \\
\hline No responde & 1 &, 8 \\
Semipresencial con aula espejo en la facultad & 19 & 15,6 \\
Semipresencial con aula espejo desde casa & 102 & 83,6 \\
Total & 122 & 100
\end{tabular}

Tabla 5. Frecuencias del tipo de semipresencialidad preferida.

La siguiente pregunta era una pregunta abierta que aborda los motivos que inclinan al estudiante hacia el aula espejo en casa o en la facultad. El análisis de esta pregunta, comentado brevemente en un apartado anterior, implicó la codificación de las respuestas abiertas. Fue posible agrupar las respuestas tal y como se refleja en la siguiente tabla (Tabla 6). 


\begin{tabular}{lrr} 
& $\mathrm{N}=122$ & $\%$ \\
\hline No responde & 9 & 4,5 \\
1. En casa mejor equipo/wifi (aula espejo en casa) & 18 & 9,0 \\
2. Evitar desplazamientos (aula espejo en casa) & 69 & 34,5 \\
3. COVID-19 (aula espejo en casa) & 21 & 10,5 \\
4. Cercanía del profesor (aula espejo en la FTI) & 7 & 3,5 \\
5. Me concentro mejor (aula espejo en la FTI/en casa) & 5 & 2,5 \\
6. La FTI debe ofrecer ambas opciones (aula espejo en casa) & 7 & 3,5 \\
7. Comodidad, conciliación, flexibilidad (aula espejo en casa) & 42 & 21,0 \\
8. Ahorro de tiempo (aula espejo en casa) & 12 & 6,0 \\
9. Feedback de compañeros (aula espejo en la FTI) & 5 & 2,5 \\
10. Otros & 5 & 2,5 \\
Total & 188 & 100
\end{tabular}

Tabla 6. Razones por las que se prefiere el aula espejo en la FTI o en casa. Tabla de frecuencias.

A continuación, se explicita brevemente la distribución de respuestas. Bajo el código 1 se incluyen todas las respuestas que hacen referencia a la mejor conexión a internet desde casa, pero también aquellas formuladas en negativo ("las aulas espejo no funcionan bien en la Facultad", "la conexión wifi en la Facultad no funciona", etc.). Bajo el paraguas de "2. Evitar desplazamientos innecesarios", se hace mención explícita a la COVID-19, pero también se habla del gasto económico y de motivos ecológicos, pero siempre incidiendo en la idea de que si no está el docente, poco importa desde dónde se visualiza la sesión retransmitida. Todas las respuestas bajo "3. COVID-19" mencionan el necesario distanciamiento social. Se hace referencia explícita a la pandemia y las recomendaciones sanitarias, a la posibilidad de evitar el contacto social y el uso de mascarillas durante tanto tiempo. Un grupo de estudiantes menciona como motivo de su preferencia del aula espejo en la Facultad la "4. Cercanía del profesor", ya que consideran que el aula espejo en la facultad abre la posibilidad de que el docente pase en algún momento y aclare dudas. Cinco estudiantes relacionan su preferencia con la facilidad para concentrarse. Algunos se concentran más en la facultad, otros en su casa. Siete estudiantes mencionan que "6. La FTI ha de ofrecer ambas posibilidades". Los estudiantes manifiestan que, aunque prefieren una modalidad desde casa, el centro debería facilitar ambas opciones para estudiantes que combinan clases presenciales con no presenciales o para aquellos con pocas posibilidades de seguir las clases desde el ámbito privado por falta de recursos o espacios adecuados. Todas las respuestas bajo el punto "7. Comodidad, conciliación y flexibilidad", argumentan que es preferible tener el aula espejo en casa porque resulta más cómodo y se pueden organizar mejor (conciliación). Bajo el punto "8. Ahorro de tiempo" se incluyen las respuestas de aquellos que prefieren el aula espejo en casa para evitar el tiempo que pierden al tenerse que desplazar hasta el centro de enseñanza. Por otro lado, los estudiantes que prefieren el aula espejo en la facultad destacan la posibilidad de recibir al menos el feedback de los propios compañeros. Estas respuestas quedan recogidas en "9. Feedback de los compañeros". En el apartado 10 se han incluido cuatro respuestas que no encajan en ninguno de los apartados anteriores: a) "es más fácil para la organización y hay que desinfectar menos aulas"; b) "porque estoy acostumbrada ya por mi experiencia en mi país de origen"; c) "siento menos ansiedad desde casa"; d) "reconoce no tener experiencia con el aula espejo en el aula".

Tal y como se aprecia en la Tabla 6, entre los que seleccionan el aula espejo desde casa, 69 sujetos $(34,5 \%)$ destacan "evitar los desplazamientos", así como motivos relacionados con 
la comodidad, la conciliación y la flexibilidad (42 sujetos, $21 \%$ ). Por otro lado, se mencionan las normas sanitarias para contener la pandemia, reducir el uso de la mascarilla (desde casa) y los problemas técnicos de conexión con la wifi desde el aula. Los únicos motivos que destacan aquellos que prefieren desplazarse a la facultad están relacionados con la interacción con los docentes (puntual, si el profesor se acerca al aula espejo) y los compañeros.

Resulta interesante que unos pocos estudiantes (7) que han seleccionado su preferencia por el aula espejo desde casa, recuerdan la necesidad de que la Facultad ponga al servicio de los estudiantes la posibilidad de seguir la docencia desde el Centro, tanto para los estudiantes sin recursos, como para aquellos que en la misma jornada de mañana o tarde alternan entre docencia presencial y no presencial, por ejemplo. Por último, en la Tabla 7 es posible ver la distribución de respuestas a la pregunta que cierra el cuestionario "¿Crees que la docencia no presencial puede sustituir a la docencia presencial en las asignaturas de interpretación?". A pesar de la clara ventaja de la docencia presencial en las preferencias de los estudiantes, una cuarta parte de los estudiantes ve posible o probable que la materia de interpretación se imparta en un formato no presencial.

\begin{tabular}{lcc} 
& Frecuencia & Porcentaje válido \\
\hline Sí & 14 & 11,5 \\
No & 80 & 65,6 \\
Tal vez & 28 & 23,0 \\
Total & 122 & 100,0
\end{tabular}

Tabla 7. Frecuencias sobre la posibilidad de sustituir la docencia presencial por la no presencial en las asignaturas de interpretación.

No sorprende que lo vean posible ya que, en la práctica, existen estudios de grado y posgrado en interpretación que se ofertan en modalidad no presencial. No obstante, este estudio no permite averiguar qué razones concretas hay detrás del $65,6 \%$ que no lo ve posible.

\section{Conclusiones}

Los estudiantes parecen identificar como gran ventaja de la docencia presencial la interacción con el profesor y el feedback que reciben en el aula. Es decir, que cualquier propuesta de semipresencialidad o no presencialidad deberá reforzar las estrategias para incluir precisamente este feedback en la propuesta.

A la vista de estos resultados, cabe recordar que los docentes no tuvieron tiempo material en el segundo semestre del curso 2019-2020 para preparar una docencia no presencial. Los grados de Traducción e Interpretación cuyos estudiantes participan en el estudio están concebidos como estudios presenciales, por lo que los docentes no tenían experiencia previa -salvo excepciones- con la docencia híbrida o no presencial. Existen experiencias en formación de interpretación no presencial que ponen de manifiesto que es posible diseñar un programa formativo que sustituya a la presencial también en esta materia. Hof (2022) destaca incluso algunas ventajas de la enseñanza no presencial de la interpretación consecutiva, como la 
posibilidad de subir las notas de consecutiva a foros donde tanto los docentes como los estudiantes puedan compartir impresiones y buenas prácticas. Si bien Hof también menciona las dificultades técnicas que algunos estudiantes no reconocen y que lastran su evolución. En ocasiones, transcurren varias semanas de docencia antes de que detecte dónde está el problema o el estudiante reconozca sus dificultades. En los últimos meses se han desarrollado aplicaciones con aplicaciones docentes en el campo de la interpretación que no estaban disponibles durante el periodo de confinamiento o que existían, pero el precio de las licencias no era asumible por los centros en aquel momento.

Por tanto, aunque estos resultados no pueden ser generalizables a los estudios que comparan los cursos presenciales con aquellos cursos o estudios diseñados para su impartición en formato híbrido o no presencial (Hodges et al., 2020), sí que refuerzan a aquellos que señalan la importancia de una preparación previa para pasar del modelo presencial a los modelos híbridos o a distancia (Boettcher y Conrad, 2016; Northrup, 2002). En definitiva, el personal docente excelente en la modalidad presencial puede serlo también en cualquier modalidad, pero no de forma automática. La falta de preparación y orientación, así como el cambio de modalidad impuesto por el estado de alarma y las normas de distanciamiento social no han sido el escenario adecuado para que este cambio fuera progresivo y formado. En el contexto concreto universitario recogido en este estudio, se organizaron cursos en línea de participación voluntaria para formar de urgencia al personal docente en algunos aspectos concretos: cómo evaluar en modalidad no presencial, la clase invertida, uso de aplicaciones de videoconferencia. Pero no se abordaron cuestiones que supusieran adaptaciones curriculares a las nuevas modalidades de enseñanza. Se entendían como medidas de emergencia. De ahí que no sea prudente extraer conclusiones sobre la enseñanza híbrida o no presencial.

Es probable que la enseñanza virtual o en línea siga ganando presencia, más allá del contexto pandémico. Las habilidades adquiridas, los materiales desarrollados por los profesores, el uso de las tecnologías digitales incorporadas a la enseñanza, han allanado el camino para la incorporación de la enseñanza combinada, semipresencial o incluso no presencial en contextos académicos reacios. Esta realidad aún convive con titulares tales como Internet a pedales en la era virtual (Canarias7, 9 junio de 2020) y otros muchos similares. Es algo que las autoridades competentes, tanto en el ámbito universitario, como en los gobiernos locales y nacionales, no pueden ignorar. Como tampoco se puede obviar que las conexiones wifi de los centros universitarios no sean eficientes para soportar la carga de la conexión simultánea de cientos de usuarios, entorpeciendo el uso de aulas espejo, por ejemplo. Las inversiones en las infraestructuras necesarias: red de datos, ordenadores, micrófonos, webcams, sistemas de audio en el aula, etc., así como estaciones de carga para los dispositivos individuales deberían considerarse prioritarias para poder adecuar los espacios para la semipresencialidad.

Otro aspecto que se debe tener presente es que la interpretación remota ya era una opción profesional antes de la pandemia, pero en estos últimos años ha ganado un terreno que no va a ceder. En este sentido, la formación híbrida y no presencial preparan al estudiante para una salida profesional real que tiene la ventaja de no depender del lugar de residencia y que con toda probabilidad represente el futuro (Song, 2022).

Como ocurre con cualquier estudio que limite los resultados a una sola institución o facultad, los resultados no son generalizables para el resto de las instituciones o facultades. La enseñanza de las asignaturas de la materia de interpretación requiere de unos laboratorios con cabinas, por ejemplo, como ya se ha mencionado, para su práctica y depende mucho de la interacción comunicativa, por lo que el riesgo percibido por la exposición al virus, podría no 
superar el inconveniente que les plantea cursar estas asignaturas en modalidades híbridas o a distancia. De ahí, la necesidad de duplicar este estudio para evaluar las posibles diferencias entre poblaciones de estudiantes, y no solo en el territorio nacional, sino también buscar las posibles diferencias con estudiantes de diferentes nacionalidades. Además, es posible diseñar un programa que ya tenga prevista la modalidad híbrida o no presencial y que se apoye en las nuevas herramientas que ofrecen solucionas a las necesidades de interrelación con los docentes y los compañeros, por ejemplo. Esta necesidad de interrelación también puede venir marcada por las culturas de origen de la muestra, de ahí el posible interés en comparar muestras de diferentes contextos culturales. Además, sería conveniente incluir no solo a estudiantes de grado, sino también a estudiantes de máster, por ejemplo, y observar si hay diferencias. Cabe esperar que las haya, puesto que las necesidades de conciliación podrían ser mayores a mayor edad. Por otro lado, cabe incluir otras variables y centrarse en aspectos como la influencia de la modalidad de la docencia en la motivación del estudiante o la capacidad de autorregulación del mismo.

\section{Referencias}

Area-Moreira, M, Bethencourt-Aguilar, A. y Martín-Gómez, S. (2020). De la enseñanza semipresencial a la enseñanza online en tiempos de Covid19. Visiones del alumnado. Campus Virtuales, 9(2), 35-50. https://bit.ly/3qrF2gQ

Arıf, S., y Omar, I. (2019). Effectiveness of flipped classroom in teaching basic English courses. Yüksekgretim Dergisi, 9(3), 279-289. https://doi.org/hcsb

Baigorri Jalón, J. (2000). La interpretación de conferencias: el nacimiento de una profesión. De París a Núremberg. Comares.

Bao, W. (2020). COVID-19 and online teaching in higher education: a case study of Peking University. Human Behavior and Emerging Technologies, 2(2), 113-115. https://doi.org/ghc588

Barak, M., Hussein-Farraj, R. y Dori, Y.J. (2016). On-campus or online: examining self-regulation and cognitive transfer skills in different learning settings. International Journal of Educational Technology in Higher Education, 13(35), 1-18. https://doi.org/hesc

Bennett, S. y Lockyer, L. (2004). Becoming an online teacher: Adapting to a changed environment for teaching and learning in higher education. Educational Media International, 41(3), 231-244. https://doi.org/brpgqv

Boettcher, J. V. y Conrad, R.-M. (2016). The online teaching survival guide. Simple and practical pedagogical tips (2. ${ }^{\mathrm{a}}$ ed.). Jossey-Bass.

Boston, W. E, Ice, P. y Gibson, A. M. (2011). Comprehensive assessment of student retention in online learning environments. Online Journal of Distance Learning Administration IV, 1. https://bit.ly/3qsOqRz

Bowers, J. y P. Kumar (2015). Students' perceptions of teaching and social presence: A comparative analysis of face-to-face and online learning environments. International Journal of Web-Based Learning and Teaching Technologies, 10(1), 27-44. https://doi.org/d83r

Brown, J. D. (2001). Using surveys in language programs. Cambridge University Press.

Caron, R. M. (2013). Teaching epidemiology in the digital age: considerations for academicians and their students. Annals of Epidemiology, 23, 576-579. https://doi.org/gm4qb9

Crawford, J., Butler-Henderson K., Rudolph, J., Malkawi, B., Glowatz, M., Burton, R., Magni, P. A. y Lam, S. (2020) COVID-19: 20 countries' higher education intra-period digital pedagogy responses. Journal of Applied Learning \& Teaching, 3(1), 9-28. https:// doi.org/ggrksc 
Cronje, J. C. (2020). Towards a new definition of blended learning. The Electronic Journal of e-Learning, 18(2), 114-121. https://doi.org/hesf

Dewaele, J-M. (2018). Online questionnaires. En A. Phakiti, P. De Costa, L. Plonsky, y S. Starfield (Eds.), The Palgrave handbook of applied linguistics research methodology (pp. 269-286). Palgrave. https://doi.org/hesg

Dörnyei, Z. (2018). Research methods in applied linguistics. Oxford University Press.

El Mansour, B. y Mupinga, D.M. (2007). Students' positive and negative experiences in hybrid and online classes. College Student Journal, 41(1), 242-248. https://cutt.ly/4EFjtQZ

Eldredge, J. (s.f.). STEM faculty blundering through remote teaching in a pandemic [Facebook page]. Facebook. Recuperado el 1 de octubre de 2021, desde https://bit.ly/3Fs587W

El País Economía. (2020, 3 de noviembre). La pandemia expone la falta de conectividad del entorno rural. CincoDías. https://bit.ly/3vbEv5L

Flores, M. A. y Gago, M. (2020). Teacher education in times of COVID-19 pandemic in Portugal: national, institutional and pedagogical responses. Journal of Education for Teaching, 46(4), 507-516. https://doi.org/gk6424

Florido, G. (2020, 9 de junio). Internet a pedales en la era virtual. Canarias7. https://bit.ly/3rl2TOI

García-Planas, M. I. y Taberna Torres, J. (2021). Transición de la docencia presencial a la no presencial en la UPC durante la pandemia del COVID-19. International Journal of Educational Research and Innovation, 15, 177-187. https://doi.org/10.46661/ijeri.5015

Graham, C. R. (2006). Blended learning systems. En C. J. Bonk y C. R. Graham (Eds.), The handbook of blended learning: Global perspectives, local designs (pp. 3-21). Pfeiffer/John Wiley and Sons, Inc.

Hodges, C., Moore, S., Lockee, B., Trust, T., y Bond, A. (2020, 27 de marzo). The difference between emergency remote teaching and online learning. Educause. https://bit.ly/3qsUKZl

Hof, Michelle (2022, 10-12 de enero). Teaching consecutive online [entrevista]. TerpSummit 2022: Confidence Camp. https://terpsummit.com/

Jaggars, S. S. (2014). Choosing between online and face-to-face courses: community college student voices. American Journal of Distance Education, 28(1), 27-38. https://doi.org/fxg8

Jaggars, S. S. (2021). Introduction to the special issue on the COVID-19 emergency transition to remote learning. Online Learning Journal, 25(1), 1-7. https://doi.org/hcsh

Karalis, T. y Raikou, N. (2020). Teaching at the times of Covid-19: inferences and implications for higher education pedagogy. International Journal of Academic Research in Business and Social Sciences, 1O(5), 479-493. https://doi.org/gktj2c

Knowles, M.S., Holton III, E.F., Swanson, R. A. (2005). The adult learner. The definitive classic in adult education and human resource development (6. ${ }^{\mathrm{a}}$ ed.). Elsevier Butterworth Heinemann.

Kurz, I. (1985). The rock tombs of the princes of Elephantine. Earliest references to interpretation in pharaonic Egypt. Babel, 21(4), 213-218. https://doi.org/cktm7c

Kurz, I. (1986). Dolmetschen im alten Rom. Babel, 32(4), 215-220. https://doi.org/bkwgpj

Lorenzetti, J. P. (2004). For quality and cost effectiveness, build a hybrid program. Distance Education Report, 8(21), 1-2, 7. https://bit.ly/3zZqoRa

Llewellyn Smith, S. (s.f.). Interpreter trainers blundering through online teaching in a pandemic [Facebook page]. Facebook. Recuperado el 1 de octubre de 2021, desde https://bit.ly/3I6C9bn

Llorens Largo, F. (2020, marzo). Recomendaciones para una docencia no presencial y apoyada con tecnología. Blog personal sobre universidad, tecnología e innovación educativa. https://bit.ly/34kYXS5

Maese, G. (2020, 30 de septiembre). Los vecinos de Cenero claman por mejoras en la red wifi y el transporte. El Comercio. https://bit.ly/3K9OEVr 
Marsh, H. W. (2001). Distinguishing between good (useful) and bad workloads on students' evaluations of teaching. American Educational Research Journal, 38(1), 183-212. https://doi.org/bcbjt3

Martínez, V. (2017). Educación presencial versus educación a distancia. La Cuestión Universitaria, 9, 108-116. https://bit.ly/3ImvGtd

Meulenbroeks, R. (2020). Suddenly fully online: a case study of a blended university course moving online during the Covid-19 pandemic. Helyon, 6(12). https://doi.org/hesj

Northrup, P. T. (2002). Online learners' preferences for interaction. The Quarterly Review of Distance Education, 3(2), 219-226.

Plota, D. y Karalis, T. (2019). Organization and implementation of a flipped classroom course in the Greek University context. Educational Journal of the University of Patras UNESCO Chair, 6(2), 53-61. https://doi.org/hesk

Popa, D., Repanovici, A., Lupu, D., Norel, M., y Coman, C. (2020). Using mixed methods to understand teaching and learning in COVID 19 times. Sustainability, 12(20), 8726. https://doi.org/gmtpvw

Romero Andonegui, A., Bilbao Quintana, N., Tejada Garitano, E. y López de la Serna, A. (2021). Diversidad de uso tecnológico en el alumnado universitario; lo académico vs. lo personal. Innoeduca. International Journal of Technology and Educational Innovation, 7(1), 19-30. https://doi.org/hesn

Rothman, E. N. (2015). Jeunes de langues. En F. Pöchhacker (Ed.), (Eds.), Routledge encyclopedia of interpreting studies (pp. 217-220). Routledge. https://doi.org/hcsp

Silió, E. (2020, 23 de abril). Los rectores estiman que 36.000 universitarios tienen trabas técnicas para seguir las clases o examinarse. El País. https://bit.ly/3qsU3iF

Song, B. (2022, 10-12 de enero). Metaverse and its impact on interpreting industry [conferencia]. TerpSummit 2022: Confidence Camp. https://terpsummit.com/

Sorokova, M. G. (2020). E-course as blended learning digital educational resource in university. Psychological Science and Education, 25(1), 36-50. https://doi.org/hcsq

Sousa Santos, S., Peset Gonzalez, M. J. y Muñoz Sepúlveda, J. (2017). La metodología flipped classroom en la enseñanza híbrida universitaria: la satisfacción de los estudiantes. IV Congreso Internacional sobre Aprendizaje, Innovación y Competitividad. https://doi.org/hesr

Universitat Oberta de Catalunya. (s.f.). Docencia no presencial de emergencia. https://bit.ly/33AzE1W

Vicioso, J. (2020, 7 de mayo). El futuro de las universidades españolas: la revolución digital ha venido para quedarse. El Mundo. https://bit.ly/351zxNY

Vladova, G., Ullrich, A. y Bender, B. (2021). Chancen und Grenzen digitaler Lehre and Hochschulen aus Studierendenperspektive: Empirische Befunde und Gestaltungshinweise. HMD. https://doi.org/hess

Wang, C., Hsu, H-C.K., Bonem, E. M., Moss, J.D, Yu, S., Nelson, D. B., Levesque-Bristol, C. (2019). Need satisfaction and need dissatisfaction: a comparative study of online and face-to-face learning contexts. Computers in Human Behavior, 95, 114-125. https://doi.org/ghvzjq

Welch, A. y Napoleon, L. (2015). Professional teaching dispositions of online instructors: why they matter. Procedia - Social Behavioral Sciences, 171, 584-589. https://doi.org/d3f4

Young, S. (2006). Student views of effective online teaching in higher education. The American Journal of Distance Education, 20(2), 65-77. https://doi.org/fsnfwz

Zawacki-Richter, O. (2020). The current state and impact of Covid-19 on digital higher education in Germany. Human Behavior and Emerging Technologies, 3(1), 218-226. https://doi.org/gnfpgg 